\title{
The sleep apnoea/hypopnoea syndrome depresses waking vagal tone independent of sympathetic activation
}

\author{
M.F. Hilton*,+,§, M.J. Chappell*, W.A. Bartlett ${ }^{\#}$, A. Malhotra ${ }^{\S}$, J.M. Beattie ${ }^{\natural}$, R.M. Cayton ${ }^{+}$
}

\begin{abstract}
The sleep apnoealhypopnoea syndrome depresses waking vagal tone independent of sympathetic activation. M.F. Hilton, M.J. Chappell, W.A. Bartlett, A. Malhotra, J.M. Beattie, R. M. Cayton. C ERS Journals Ltd 2001.

ABSTRACT: The modest daytime hypertension and sympathetic upregulation associated with the sleep apnoea/hypopnoea syndrome (SAHS), does not explain the relatively large increased risk of cardiac morbidity and mortality in the SAHS patients population. Therefore, efferent vagal and sympathetic activity was evaluated during wakefulness in SAHS subjects and matched healthy controls, in order to determine if vagal downregulation may play a role in the aetiology of cardiac disease in the SAHS.

The awake autonomic nervous system function of 15 male subjects, with mild-tomoderate SAHS was compared to that of 14 healthy controls matched for age, body mass index, gender and blood pressure. All subjects were free from comorbidity. Vagal activity was estimated from measurements of heart rate variability high frequency power (HF) and sympathetic activity was measured from urine catecholamine excretion.
\end{abstract}

The $\% \mathrm{HF}$ power was significantly $(\mathrm{p}<0.03)$ reduced in SAHS patients $(10 \pm 1.6$ $($ mean \pm SEM)) as compared to controls $(17 \pm 3)$. In addition, HF power correlated with the apnoea/hypopnoea index in the SAHS subjects $(R=-\mathbf{0 . 5 9 2}, p=0.02)$. There was no statistically significant difference in the daytime excretion of nonadrenaline between control $\left(242 \pm 30 \mathrm{nmol}^{-c^{2} \text { collection }}{ }^{-1}\right)$ and SAHS $\left(316 \pm 46 \mathrm{nmol}^{\circ}\right.$ collection $\left.^{-1}\right)$ subjects $(\mathbf{p}=\mathbf{0 . 3 8})$.

In these sleep apnoea/hypopnoea syndrome patients there was limited evidence of increased waking levels of urine catecholamines. The principal component altering waking autonomic nervous system function, in the sleep apnoea/hypopnoea syndrome subjects, was a reduced daytime efferent vagal tone.

Eur Respir J 2001; 17: 1258-1266.
*School of Engineering, University of Warwick, Coventry, UK, Depts of ${ }^{\#}$ Clinical Biochemistry, Cardiology, and ${ }^{+}$Respiratory Medicine, Birmingham Heartlands Hospital, Bordesley Green East, Birmingham, UK, 'Sleep Disorders Program, Circadian, Neuroendocrine and Sleep Disorders Section, Brigham and Women's Hospital/ Harvard Medical School, Boston, MA, USA

Correspondence: M. Hilton, Circadian, Neuroendocrine and Sleep Disorders Section, Sleep Disorders Program, Brigham and Women's Hospital, RFB-486, 221 Longwood Avenue, Boston, MA, 02115 USA. Fax: 16179750809

\section{Keywords: Apnoea}

autonomic nervous system

heart

heart rate variability

sympathetic

vagus

Received: February 12000

Accepted after revision March 152001

This work was supported by the Mathematics in Medicine Initiative (MiMl) from the University of Warwick and the British Medical Association, Board of Science and Education, Edith Walsh and Ivy Powell Research Grant Award.
Persuasive evidence indicates that waking sympathetic neural traffic is elevated in sleep apnoea/ hypopnoea syndrome (SAHS) patients as compared with healthy individuals $[1-7]$.

This may contribute to the increased prevelance of hypertension in the SAHS [2]. However, the modest daytime levels of hypertension (reviewed in [8]) reported in the SAHS are unlikely to be completely responsible for the reported striking increased risk of cardiac morbidity and mortality $[9,10]$. A limitation of studies that examine only the sympathetic system is that they do not necessarily imply parasympathetic (vagus) tone or describe an autonomic balance. The vagus and sympathetic systems may have separate controllers that allow independent changes in activity in a homeostatic state $[11,12]$. Withdrawal of vagal efferent activity from the myocardium is known to have adverse cardiovascular consequences [13-16]. Thus, efferent vagal and sympathetic activity were evaluated during wakefulness in SAHS subjects and matched healthy controls in order to determine whether vagal downregulation plays a role in the aetiology of cardiac disease in the SAHS.

Power spectral analysis of heart rate variability (HRV) offers a noninvasive quantification of efferent autonomic tone. The high frequency (HF) component of the HRV power spectrum is universally regarded as a reliable indicator of efferent vagal activity resulting from respiratory sinus arrhythmia $[17-20]$. Power in the low frequency (LF) band is traditionally associated with sympathetic activity primarily from Mayer waves [21, 22]; however, it may have some vagal input $[17,23]$. The LF:HF ratio provides a measure of sympatho-vagal balance [20, 24]. Recently, HRV 
studies have quantified daytime sympathetic and vagal tone in SAHS subjects with conflicting conclusions [7, $25-30]$. This is most likely due to methodological differences such as: 1) ambulatory long-term studies versus short-term resting studies; 2) inclusion of subjects with comorbidity; or 3) lack of matched controls. In addition, the interpretation of HRV sympathetic measures may require careful consideration [31]. The majority of previous studies utilizing HRV in the SAHS have not "benchmarked" sympathetic markers with an alternative measure. Thus, the autonomic profile of SAHS subjects during the waking hours is poorly defined.

The principal that autonomic dysfunction during wakefulness in SAHS patients is sympathetically or parasympathetically mediated was tested. To address previous limitations, the daytime (waking) autonomic function of SAHS subjects (without known comorbidity) was compared with that of matched healthy controls. The HF component of the HRV power spectrum was applied to evaluate cardiac efferent vagal activity. Sympathetic activity was measured as the daytime excretion of urine catecholamines and was compared to the HRV sympathetic marker of LF power. Cardiovascular reflex testing was used as a global measure for the identification of clinically overt autonomic dysfunction.

\section{Methods}

\section{Subjects}

Fifteen male SAHS subjects, referred consecutively to the Sleep Disorders Unit, Birmingham Heartlands Hospital, UK, were recruited. Fourteen healthy male controls were recruited from local radio and informative newspaper advertisements. The diagnosis of the SAHS was established by overnight in-laboratory polysomnography, defined as an apnoea/hypopnoea index (AHI) $>15 \mathrm{~h}^{-1}$, together with associated symptoms including snoring, unrefreshing sleep or excessive daytime sleepiness. The control population was defined as having an AHI of $<10 \mathrm{~h}^{-1}$ with no symptoms of excessive daytime sleepiness. The local Ethics Committee approved the study protocol. Written informed consent was obtained from each subject prior to participation in the study. Inclusion criteria included: no history of cardiovascular disease, age 25-69 yrs, and normal lung function (forced expiratory volume in one second (FEV1) and forced vital capacity (FVC) $>80 \%$ predicted). Exclusion criteria included: shift-work, autonomic neuropathy, diabetes, asthma, thyroid disease, diagnosed hypertension, any medication that may affect autonomic function or any sleep pathology other than SAHS. Anthropomorphic details of the subject populations are shown in table 1.

\section{Protocol}

Subjects were asked to avoid consuming alcohol or caffeinated beverages for $24 \mathrm{~h}$ before, or during the study. Participants underwent overnight polysomnography in a dedicated sleep laboratory. On final awakening they evacuated the bladder. All urine passed between the hours of final wakening and lights out on the following evening $(22: 00-23: 00 \mathrm{~h})$ was collected for subsequent urine catecholamine analysis. Starting at 10:00 h, electrocardiogram (ECG) recordings were performed with the subjects awake and their eyes open over 30-min postural periods of: 1) resting supine; and 2) standing still. To ensure wakefulness, subjects were monitored via a remote video link. Following this cardiovascular reflex tests [32] of the Valsalva manoeuvre, lying to standing blood pressure and heart rate changes were performed.

\section{Cardiovascular reflex tests}

After a minimum of 15-min supine, the subjects' systolic and diastolic blood pressures were measured via an arm cuff sphygmomanometer, following which they were asked to stand without assistance. Blood pressure measurements were repeated 1 min after standing. The difference in the systolic pressures between supine and standing was taken as the measure of postural blood pressure change [32]. During the same supine to standing period, the heart rate response to standing was calculated as the ratio of the longest ECG R-R interval around the 30 th beat to

Table 1.-Comparison of anthropomorphic and polysomnography results

\begin{tabular}{|c|c|c|c|c|c|}
\hline & \multicolumn{2}{|c|}{ Controls } & \multicolumn{2}{|c|}{ SAHS } & \multirow[t]{2}{*}{ p-value } \\
\hline & Mean \pm SEM & Range & Mean \pm SEM & Range & \\
\hline Age yrs & $43.8 \pm 2.5$ & $29-60$ & $47.2 \pm 1.8$ & $34-56$ & 0.246 \\
\hline $\mathrm{BMI} \mathrm{kgm}^{-2}$ & $28.4 \pm 0.9$ & $23-36$ & $32.9 \pm 1.7$ & $23-44$ & 0.077 \\
\hline Systolic BP mmHg & $112 \pm 4$ & $88-128$ & $127 \pm 5$ & $85-158$ & 0.053 \\
\hline Diastolic BP mmHg & $75 \pm 3$ & $60-98$ & $83 \pm 3$ & $60-102$ & 0.943 \\
\hline $\mathrm{AHI} \mathrm{h}^{-1}$ & $4 \pm 0.9$ & $0-8$ & $45 \pm 7$ & $17-105$ & $<0.001$ \\
\hline Arousal Index $\mathrm{h}^{-1}$ & $23 \pm 2.8$ & $8-40$ & $49 \pm 5.0$ & $29-94$ & $<0.001$ \\
\hline Lowest $\mathrm{Sa}_{\mathrm{a}, \mathrm{O}_{2}} \%$ & $87 \pm 1.0$ & $80-92$ & $80 \pm 3.0$ & $47-90$ & 0.045 \\
\hline Average $S_{\mathrm{a}}, \mathrm{O}_{2}$ desaturation $\%$ & $1.5 \pm 0.3$ & $0-3$ & $5.6 \pm 1.6$ & $1-23$ & 0.03 \\
\hline Epworth sleepiness score & $6 \pm 0.5$ & $3-9$ & $14 \pm 1.6$ & $4-24$ & 0.016 \\
\hline
\end{tabular}

SAHS: sleep apnoea/hypopnoea syndrome; BMI: body mass index; BP: blood pressure; AHI: apnoea/hypopnoea index; $S_{\mathrm{a}, \mathrm{O}_{2}}$ : arterial oxygen saturation. 
the shortest $\mathrm{R}-\mathrm{R}$ interval around the 15 th beat after standing $[32,33]$.

Prior to performing the Valsalva manoeuvre, subjects were sat quietly for a period of $5 \mathrm{~min}$. The manoeuvre was performed by blowing into an occluded mouthpiece (glottis open) maintaining an oesophageal pressure of $40 \mathrm{mmHg}$ for $20 \mathrm{~s}$ [34], after which the mouthpiece was removed and the subjects' respiration returned to normal for the following $40 \mathrm{~s}$. The Valsalva ratio was calculated as the ratio of the longest $\mathrm{R}-\mathrm{R}$ interval after the manoeuvre to the shortest R-R interval during the manoeuvre from the average of three technically successful manoeuvres $[32,34]$.

\section{Holter electrocardiogram and heart rate variability assessment}

A two channel Holter monitor (Oxford Medilog MR-45, Oxford Instruments, Abingdon, UK) was used to record the ECG $\left(\mathrm{CM}_{5}\right.$ and $\mathrm{CC}_{5}$ leads). A trained observer edited the ECG. Only normal to normal R-R intervals were used for subsequent analysis. All ECG records had $<5 \%$ ectopy. The first 5 min of supine and standing postural states were considered to be stabilization periods and were not included in the HRV analysis. The subsequent $25 \mathrm{~min}$ section of data was used for analysis.

Calculation of HRV was standardized according to published criteria [20]. Prior to calculation of frequency domain indices, the nonequispaced R-R tachogram was interpolated with a cubic spline and resampled at $3.413 \mathrm{~Hz}$ developing a 1024 point equispaced time series per 5-min segment. Preprocessing by removing the least squares linear regression approximated stationarity in the signal. The power spectral density was calculated using WeLCH's [35] technique for the fast Fourier transform with a Hanning window length of 512 points and an overlap of 256 points. Spectral powers, in units of $\mathrm{ms}^{2}$, were calculated for the frequency bands of: 1) HF, $0.15-0.40 \mathrm{~Hz}$; 2) LF, $0.04-0.15 \mathrm{~Hz}$; and 3) total power (TP), $0-0.40 \mathrm{~Hz}$. Calculated power spectral indices included: per cent HF $(\% \mathrm{HF})$, per cent LF (\%LF), and the LF/HF ratio.

\section{Urine catecholamines}

Urine was collected in $2.5 \mathrm{~L}$ polyethylene containers containing $50 \mathrm{~mL}$ of $3 \mathrm{~mol} \cdot \mathrm{L}^{-1} \mathrm{HCl}$ solution. Urinary unconjugated (free) catecholamines were measured using isocratic reversed-phase ion-paired electrochemical detection with multiple electrode reductive mode electrochemical detection. Automated ion-exchange extraction of the catecholamines from the urine specimens was carried out using the Gilson ASTED system (Anachem, Bedfordshire, UK) as described by GreEn et al. [36]. The detection system employed a Coulochem 5200A with 5011 incorporating a 5021 conditioning cell and 5011 high sensitivity amperometric analytical cell (ESA Inc., Chelmsford, MA, USA). The conditioning cell was held at $+0.35 V$. A screening potential of $-0.05 V$ was applied to the first electrode in the analytical cell and the catecholamines were detected following reduction at $-0.35 \mathrm{~V}$ at the second downstream electrode. The urinary outputs of free noradrenaline and adrenaline were calculated in terms of nmol-period of collection ${ }^{-1}$.

\section{Polysomnography}

Polysomnographic (Compumedics S-Series Sleep System, version 5, Compumedics Ltd, Victoria, Australia) measurements included: bipolar ECG, chest and abdominal wall expansion, body position, oro-nasal airflow (thermistor), leg movement (piezoelectric sensors), snoring, oximetry (Minolta Pulsox-7 Oximeter, AVL Medical Instruments Ltd, Schaffhausen, Switzerland), two channel electroencephalogram (EEG) $\left(\mathrm{A}_{1}-\mathrm{C}_{3}\right.$ and $\left.\mathrm{A}_{2}-\mathrm{C}_{4}\right)$, submental electromyogram (EMG), left and right electro-oculogram (EOG). Sleep staging was performed using standard criteria [37]. Arousals were scored using the American Sleep Disorders Association (ASDA) 3-second definition [38]. A trained technician scored respiratory traces with respiratory events classified into obstructive, central or mixed apnoea or hypopnoea using published methods [39].

\section{Statistics}

Individual cardiovascular reflex results were classified as normal, equivocal or abnormal as per published guidelines [32]. Comparisons were performed using the Chi-squared statistic $\left(\chi^{2}\right)$ on the results grouped by individual tests and the overall sum of tests. An unpaired parametric t-test was used to evaluate inter-group differences in actual cardiovascular reflex test results. To create a normal distribution prior to statistical comparisons, HRV absolute powers of TP, HF and LF were transformed using the natural logarithm (ln). Inter-group comparisons for urine catecholamine and HRV results were computed with a parametric t-test. To estimate variables that best predicted daytime HF power and urine catecholamine concentrations, multiple linear regressions were performed on the SAHS group. The SAHS group was subdivided into severe and mild-tomoderate categories based on an AHI threshold of $40 \mathrm{~h}^{-1}, \% \mathrm{HF}$ power and urinary noradrenaline. Thresholds for $\% \mathrm{HF}$ power and noradrenaline were decided by minimizing the Fisher exact Chi-squared analysis result. Reported probability values (p) are for a two-tailed distribution. Statistical comparisons were performed using Statistical Package for the Social Sciences (SPSS) for Windows, version 8.0 (SPSS Inc., Chicago, IL, USA).

\section{Results}

The SAHS and control subjects were well matched for age (table 1). There were no statistical differences 
$(\mathrm{p}<0.05)$ between the groups blood pressures or body mass index (BMI) values although there was a trend for the SAHS subjects to have elevated systolic pressures and BMIs. There were no historical differences in self-reported alcohol, tobacco or caffeine consumption between the two groups. Polysomnography showed a wide range of SAHS severity in terms of the AHI, desaturation and the Epworth sleepiness score (table 1). The majority of SAHS subjects had mild disease with nine of 15 having an AHI $<40$ (fig. 1a) accompanied by an average desaturation $<4 \%$ (fig. 1b). Inter-group comparisons of the cardiovascular reflex ratios (table 2) indicated no significant difference between the control and SAHS groups. Classifying results as normal, equivocal or abnormal did not reveal any further differences.

There were no significant differences between SAHS and control subjects for mean adrenaline $(p=0.43)$ or noradrenaline $(\mathrm{p}=0.8)$ during the waking period (fig. 2). The HRV results are illustrated in figure 3. While resting supine (fig. 3a), the LF:HF ratio was significantly higher in the SAHS group indicating a shift in the sympatho-vagal balance toward the sympathetic system. This shift in the LF:HF ratio was due primarily to a reduced $\% \mathrm{HF}$ power (vagus) in the SAHS as compared to the controls $(\mathrm{p}=0.029)$, as the $\%$ LF power (sympathetic) was not significantly different between the two groups $(\mathrm{p}=0.66)$.
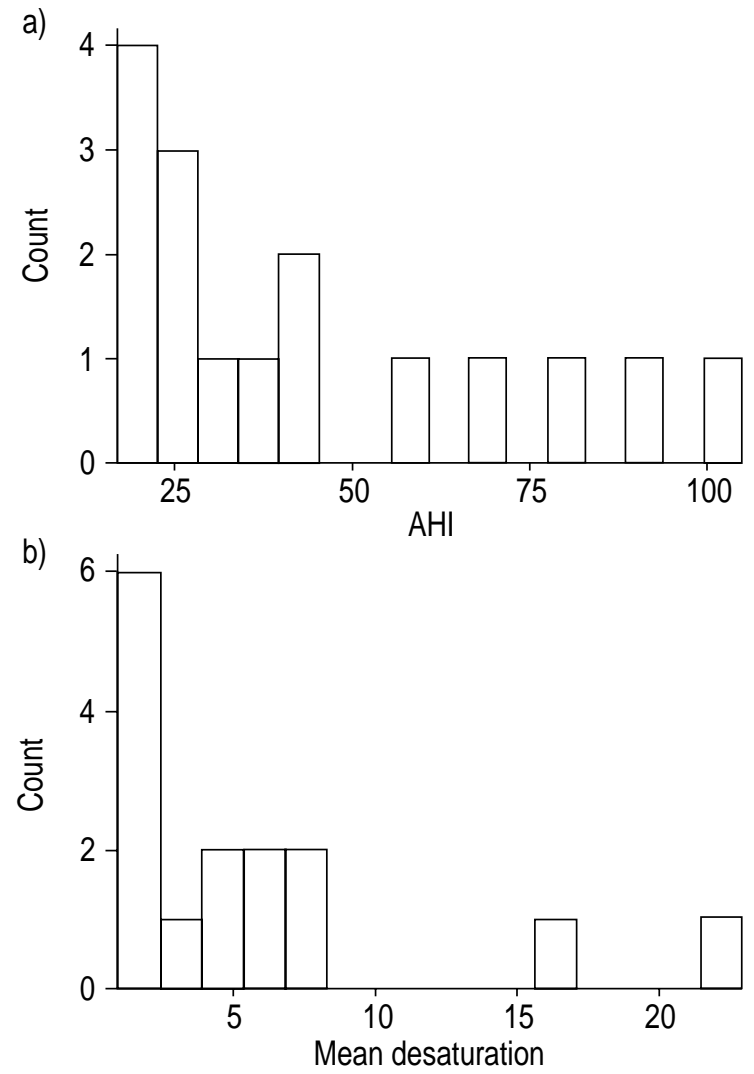

Fig. 1. - The distribution of a) apnoea/hypopnoea index (AHI) and $b$ ) the average desaturation per respiratory event for the sleep apnoea/hypopnoea syndrome group illustrating that most of the subjects had a relatively low AHI and minor desaturations i.e. $<4 \%$.
Table 2.-Inter-group comparisons of cardiovascular reflex results for heart rate $(\mathrm{HR})$ and blood pressure $(\mathrm{BP})$ response to standing and the Valsalva manoeuvre. All results are ratios and as such are unitless

\begin{tabular}{lclc}
\hline Responses & Controls & SAHS & p-value \\
\hline HR & $1.16 \pm 0.02$ & $1.13 \pm 0.04$ & 0.945 \\
BP & $-4.08 \pm 2.4$ & $2.60 \pm 3.9$ & 0.096 \\
Valsalva & $1.63 \pm 0.07$ & $1.64 \pm 0.08$ & 0.854 \\
\hline
\end{tabular}

Data are presented as mean \pm SEM. SAHS: Sleep apnoea/ hypopnoea syndrome; HR: heart rate; BP: blood pressure.

The orthostatic challenge of standing amplified the supine HRV results. During the standing period, the mean R-R period was significantly shorter in the SAHS as compared to the controls $(p=0.036)$. This was due principally to a large reduction of the $\mathrm{HF}$ power for the SAHS subjects $(p=0.004)$ while the LF power was not statistically different between SAHS and control subjects $(\mathrm{p}=0.07)$. The total power was also significantly lower for the SAHS subjects on standing.

Neither age nor BMI correlated significantly with awake urinary catecholamine concentrations in the SAHS group $(\mathrm{R}=-0.408, \mathrm{p}=0.131$ and $\mathrm{R}=0.439$, $\mathrm{p}=0.101$, respectively). Iterative multiple linear regression modelling, using forward, backward, stepwise, and removal techniques, was performed with
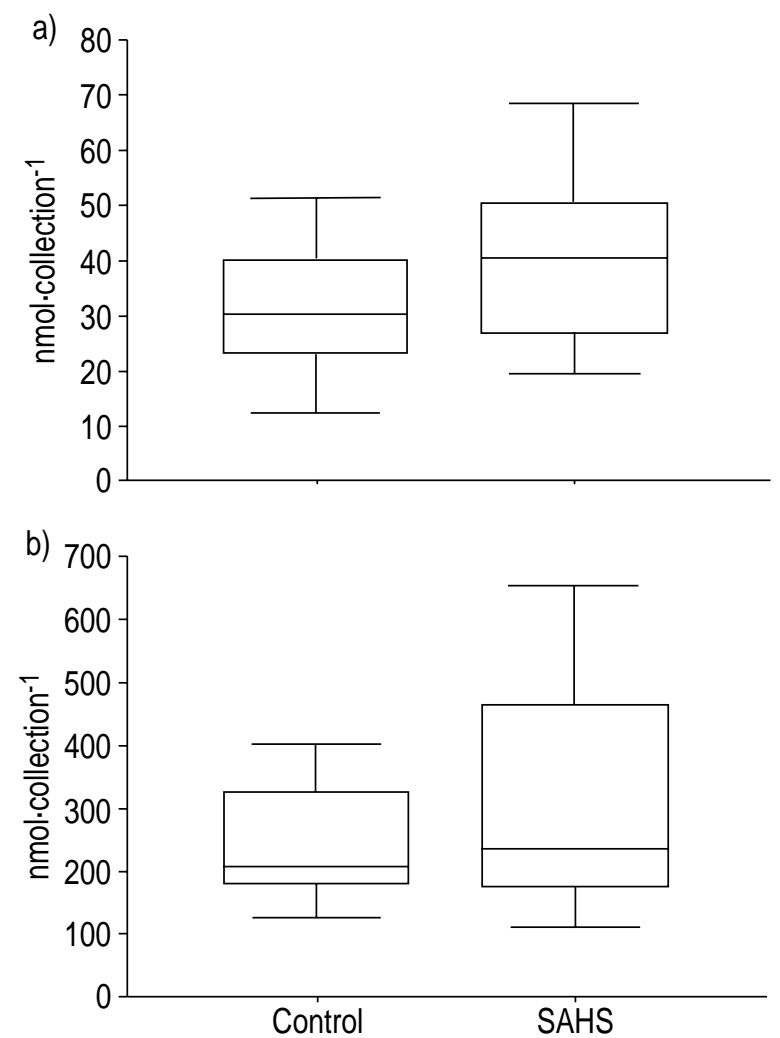

Fig. 2. - Daytime urine concentrations of a) adrenaline and b) noradrenaline in sleep apnoea/hypopnoea syndrome (SAHS) and control subjects. Boxes represent 1 quartile above and below the mean values (line in the box) and the whiskers illustrate the $95 \%$ confidence intervals of the data. 

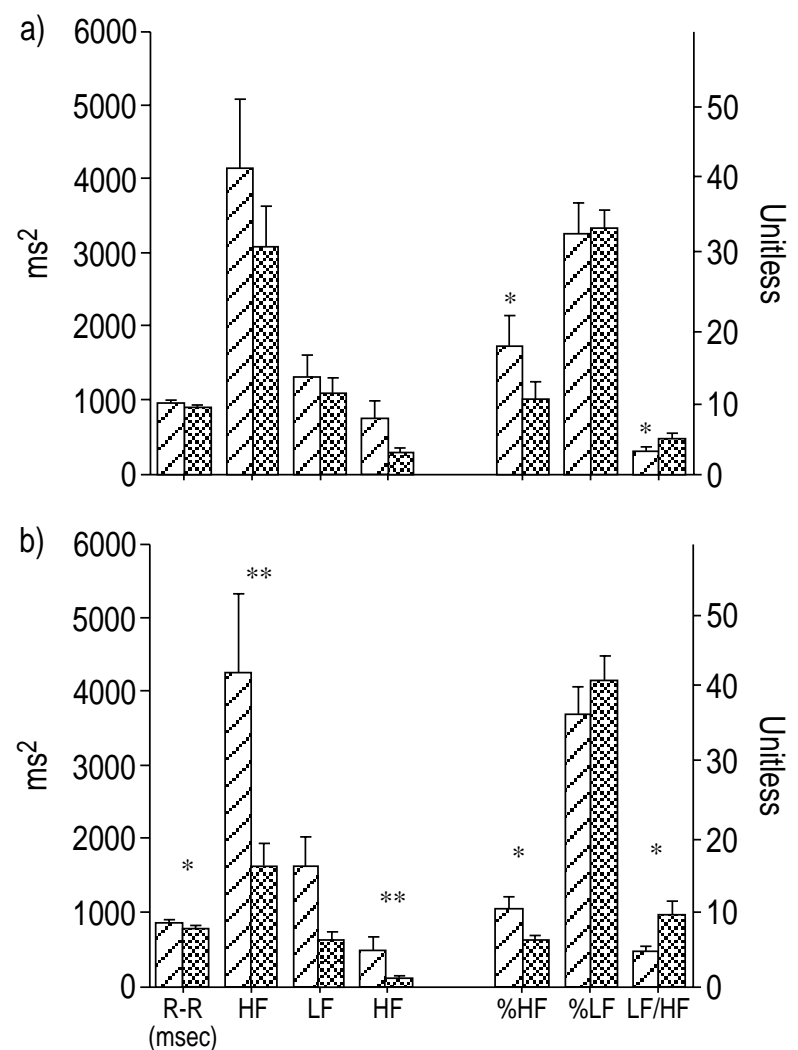

Fig. 3. - Heart rate variability results for the sleep apnoea/hypopnoea syndrome (网) (SAHS) and control groups (区্Z) during postural states of a) supine and b) standing. TP: total power; LF: low frequency; HF: high frequency. ${ }^{*}: \mathrm{p}<0.05 ; * *: \mathrm{p}<0.01$ for comparisons between control and SAHS subjects.

noradrenaline as the dependent variable and polysomnographic indices of AHI, arousal index, average arterial oxygen saturation $\mathrm{Sa}, \mathrm{O}_{2}$ desaturation per respiratory event (event $\mathrm{S}_{\mathrm{a}}, \mathrm{O}_{2}$ ), mean overnight $\left(\mathrm{Sa}, \mathrm{O}_{2}\right)$, and lowest overnight $\mathrm{Sa}, \mathrm{O}_{2}$ as independent variables. In each model the decrease in event $S_{\mathrm{a}}, \mathrm{O}_{2}$ was identified as the strongest predictor of awake urine noradrenaline $(\mathrm{R}=0.803, \mathrm{p}=0.001)$ with other variables not significantly adding to the model prediction (fig. 4). The same multiple linear regression procedure was performed for HRV vagal indices of the natural logarithm of supine HF power (lnHF) and $\% \mathrm{HF}$ power as the dependent variables. InHF power supplied the most robust model fit. AHI was the strongest model predictor of awake supine vagal tone $(\ln H F)(\mathrm{R}=-0.592, \mathrm{p}=0.02)$ suggesting that the frequency of SAHS events is a better predictor of reduced vagal activity than oxygen desaturation in awake SAHS subjects. $\operatorname{lnHF}$ and awake noradrenaline did not significantly correlate with each other $(\mathrm{R}=-0.485, \mathrm{p}=0.067)$. When lnHF was added to the multiple linear regression models for noradrenaline and noradrenaline was likewise added to the model for InHF, neither significantly added to prediction results.

A nonlinear analysis evaluating the effects of mean oxygen desaturation and AHI on autonomic tone was performed by subdividing the SAHS subjects into

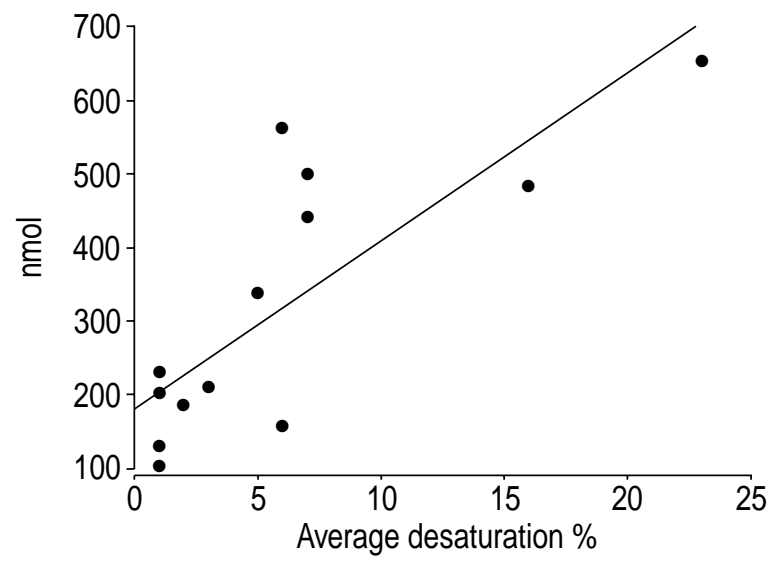

Fig. 4. - Scatter plot of average arterial desaturation associated with a respiratory event versus daytime noradrenaline concentrations with the least squared regression prediction line. $\mathrm{R}=0.803$; $\mathrm{p}=0.001$.

nine mild $\left(\mathrm{AHI} \leq 40^{-1}\right)$ and six moderate-to-severe $\left(\mathrm{AHI}>40 \mathrm{hr}^{-1}\right)$ subjects. SAHS subjects were then classified into quadrants based on high and low awake urine noradrenaline concentrations and supine $\% \mathrm{HF}$ power (fig. 5). It is evident that in the moderate-tosevere SAHS group (fig. 5) all subjects had both elevated urine noradrenaline concentrations and low $\%$ HF power (vagal tone) values. $\%$ HF power and urine noradrenaline performed comparably in differentiating SAHS disease severity (Chi-squared: $\mathrm{p}<0.002$ versus $\mathrm{p}<0.03$, respectively). Comparing the mild SAHS group (AHI $<40 \mathrm{~h}^{-1}$ ) to controls there were no statistical differences in supine $\% \mathrm{HF}$ power $(\mathrm{p}=0.27)$ or waking urine noradrenaline $(\mathrm{p}=0.64)$. However, standing \%HF was lower in the mild SAHS group as compared with controls $(\mathrm{p}<0.04)$ with no significant differences in $\% \mathrm{LF}(\mathrm{p}=0.49)$.

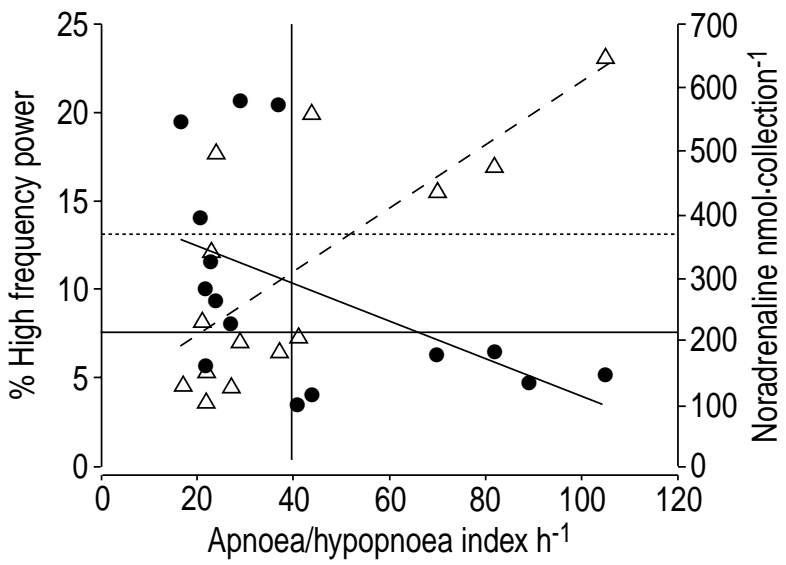

Fig. 5. - Scatter plot of awake supine per cent high frequency $(\% \mathrm{HF})$ power $(\boldsymbol{O})$ and waking urinary noradrenaline excretion $(\triangle)$ versus the apnoea/hypopnoea index per hour with the least squared regression prediction lines. The vertical line at an $\mathrm{AHI}=40 \mathrm{~h}^{-\mathrm{T}}$ divides the sleep apnoea/hypopnoea syndrome subjects into mild and severe groups. The horizontal line $(-)$ at $\% \mathrm{HF}=7.5$ and the dashed horizontal line $(\cdots \cdot)$ at noradrenaline $=$ 390 nmol divides the subjects into high and low vagal and sympathetic categories respectively. 


\section{Discussion}

A clear reduction in myocardial vagal tone was found in the SAHS group as compared to controls. This reduction in vagal tone in SAHS subjects occurred in the absence of an overall mean sympathetic upregulation or gross cardiac dysfunction as indicated by cardiovascular reflex tests. In the SAHS subjects, AHI was the best predictor of waking vagal tone. The majority of SAHS subjects were at the mild end of the disease spectrum with only minor nocturnal desaturations. However, nocturnal desaturation was significantly correlated with increasing waking noradrenaline concentrations. This indicates that, in desaturating SAHS subjects (more severe disease) there is an increase in waking sympathetic activity and concomitant reduction in waking vagal tone (fig. 5). However, the frequency of SAHS events per se, in the absence of arterial oxygen desaturations (mild disease), may be principally responsible for altering efferent vagal tone disturbing the autonomic balance. It may be that, as SAHS severity increases with greater desaturations, there is both a waking sympathetic elevation as well as the ongoing reduction in vagal tone.

\section{Catecholamines}

The group mean data indicate no statistically significant difference in urine catecholamine concentrations between SAHS and healthy control subjects. This is in apparent contrast to the preponderance of evidence which suggests increased daytime sympathetic tone in SAHS patients [1, 2, 4, 5, 7, 40, 41]. However, previous studies measuring catecholamines in the SAHS differed from the current study in that they contained subjects with a higher AHI and increased nocturnal desaturations [1, 5, 41], were unmatched for hypertension or comorbidity [2, 5] or the controls were unmatched for both BMI and age [5, 41]. In a well-controlled study by NARKIEwICZ et al. [4], with patient and control groups similar to the present study, resting, muscle sympathetic nerve activity (MSNA) was elevated in awake SAHS subjects resting supine suggesting different findings from the data presented here. The sympathetic response to hypoxia was significantly higher in SAHS patients only during voluntary apnoea [4]. It may be that in ambulant mild SAHS subjects, some factors (such as blood pressure and breathing) serve to reduce or mask inter-group sympathetic differences. The results of NARKIEWICZ et al. [4] and the urine catecholamine results presented here cannot be directly compared as they were conducted in differing physiological conditions, namely controlled supine versus uncontrolled ambulatory. However, the \% $\%$ LF results while the present subjects were supine also indicate no mean increase in sympathetic activity while in the controlled supine posture. The finding suggests there may be some divergence between cardiac and peripheral (MSNA) sympathetic activity and that increases in peripheral sympathetic tone can be accompanied by increases in cardiac vagal tone [42] effectively decreasing cardiac sympathetic tone via reciprocally inhibitory effects [15], while MSNA burst frequency is not subject to peripheral vagal inhibition [43]. Thus, sympathetic measurements at different sites ie. cardiac versus MSNA burst frequency, may be measuring differing sympathetic activities. It may also be the case that, compared to MSNA measurements, $\%$ LF power does not have the sensitivity to quantify small cardiac sympathetic differences between mild SAHS and controls due to the possibility that \% $\% F$ power has inputs in addition to sympathetic activity [31].

Although there were no group mean differences, within the SAHS subjects the average event desaturation during sleep was strongly associated with waking noradrenaline levels. This finding indicates that increasing SAHS disease severity, as measured by $\mathrm{S}_{\mathrm{a}}, \mathrm{O}_{2}$ desaturation, is associated with elevated sympathetic activation. This supports animal [44-47] and human $[3,4]$ model findings that episodic nocturnal hypoxia, or a combined effect of apnoea and hypoxia, augments daytime sympathetic activity. However, in the current study, $\mathrm{Sa}_{\mathrm{a}} \mathrm{O}_{2}, \mathrm{AHI}$ and arousals were strong covariates and it is difficult to separate the precise inter-relationship. The lack of an inter-group difference in daytime noradrenaline despite the association of $\mathrm{Sa}_{2} \mathrm{O}_{2}$ with daytime noradrenaline in the SAHS patients suggests: 1) that SAHS patients with minimal desaturation may be relatively normal in terms of sympathetic activity while ambulatory; and 2) that desaturation increases waking sympathetic outflow.

\section{Heart rate variability}

In accordance with previous findings in patients with AHI $>20 \mathrm{~h}^{-1}$ [7], the SAHS subjects of the present study have a significantly higher LF:HF ratio, in both the supine and standing postures. This indicates an adjustment of the sympatho-vagal balance in these subjects. Supporting the daytime noradrenaline results, there were no statistical differences in $\% \mathrm{LF}$ between the SAHS and control subjects for either postural position, indicating that mean sympathetic activity was similar in the two populations. It has been stated that cardiac sympathetic activity from HRV measurements is reduced in SAHS subjects [7]. The HRV measurement used to examine this sympathetic effect was normalized (nu) LF power (LFnu). LFnu and HFnu divides the $\mathrm{LF} / \mathrm{HF}$ ratio into its principal components making the assumption that the vagal and sympathetic limbs are directly reciprocally related. Evidence from circadian [11] and diving reflex studies [48] suggests that the sympathetic and vagal systems do not act purely as a balance swinging around a fulcrum, but are also independently modulated. Therefore, $\% \mathrm{HF}$ and $\% \mathrm{LF}$ measures may more directly reflect vagal and sympathetic activity. In the study by NARKIEWICZ et al. [7], LFnu increased and HFnu decreased in SAHS subjects as compared to controls, due to the mathematical reciprocal nature of normalized units. The autonomic alterations in the study by NARKIEWICZ et al. [7] are similar to those reported here in that they reported no change in 
absolute LF power but a significant reduction in absolute HF power in SAHS subjects as compared to controls (per cent powers were not reported). Therefore, the principal component shifting the sympathovagal balance in the SAHS subjects was a significant reduction in efferent cardiac vagal tone (HF power) as compared to the control subjects, as is reported here. These results suggest that in the absence of group mean sympathetic alterations, there was a significant reduction in vagal efferent activity in SAHS patients. Linear regression and nonlinear quadrant analyses indicate that the degree of vagal inhibition relates to the frequency of SAHS events though $\mathrm{Sa}_{\mathrm{a}} \mathrm{O}_{2}$ desaturation is a covariate.

To date there has been little consensus on the impact of the SAHS on measures of awake HRV with reports of no differences in LF or HF [27], reduced $\mathrm{HF}$ and increased LF [26] as well as morning reductions in both HF and LF [28]. In the aforementioned studies the SAHS patients had comorbidity, controls were not matched for age and BMI and the interpretation of the sympatho-vagal balance was not compared with an alternative measure of sympathetic activity. This may be necessary as the ability of the LF power to reflect sympathetic activity is a topic of debate [31].

The present findings of a reduced HF power with no change in absolute LF power are concordant with those of NARKIEWICZ et al. [7], who compared wellmatched controls and SAHS subjects with no comorbidity. Supporting evidence for the SAHS resulting in reduced daytime vagal activity is that treatment of the SAHS with continuous positive airway pressure has been noted to increase awake HF power [26, 29] with no statistical change in absolute LF power [29].

\section{Interpretation and mechanisms}

The results presented illustrate that in the absence of a group mean increase in sympathetic activity, decreased vagal tone is present in relatively mild SAHS patients without comorbidity. It has been shown previously that during the waking hours, SAHS subjects may have a reduced baroreceptor sensitivity [40] or a resetting of baroreceptors [49]. A decrease in vagal afferents serves to decrease vagal efferent activity [15]. This is one of many possible mechanisms for a daytime decrease in vagal efferent activity. A decrease in vagal efferent activity and impaired cardiopulmonary baroreflex mediated responses are typically associated with heart failure $[50,51]$. A decrease in vagal tone may facilitate cardiac arrhythmias due to the removal of pre- and post-synaptic vagal inhibitory actions on the cardiac response to sympathetic stimulation [13-16]. Thus, a reduction in vagal tone in SAHS patients may have significant clinical implications especially during periods of sympathetic excitation.

The data presented support the hypothesis that the vagal system may be affected in mild SAHS patients, with minimal desaturation, in the absence of elevated sympathetic tone. The exact temporal succession of first a decrease in vagal tone followed by an increased sympathetic tone cannot be established definitively from the results presented due to inadequate power for such an analysis. However, it is interesting to speculate that as the natural history of SAHS may be a progression from snoring to SAHS with desaturations, so may autonomic alterations follow with: 1) vagal tone decreasing with increasing respiratory event frequency; and 2) sympathetic tone increasing with increasing oxygen desaturations. The orthostatic challenge of standing produced the most significant differences in HRV measures with the SAHS subjects having an overall reduction in total power and an exaggerated swing in the sympatho-vagal balance. In addition, the subgroup analysis showed that mild SAHS subjects (AHI $<40 \mathrm{~h}^{-1}$ ) had a lower vagal tone in the standing posture than healthy controls with no significant alteration is sympathetic tone (standing $\%$ LF or total awake urinary noradrenaline excretion). This simple manoeuvre may reveal an inability of the autonomic system in subjects with sleep apnoea to respond to physiological change which may stress the circulatory system.

\section{Limitations}

The BMI values were not significantly different between the two study groups but the trend was for the SAHS subjects to have elevated BMI. However, as in previous reports, in the current study BMI did not correlate with either urine free noradrenaline [41] or HF power such that any effect is most likely insignificant. In the current study, wakefulness was not monitored with EEG recordings and the more severe SAHS subjects, who found it difficult to maintain wakefulness in the supine posture, were on occasion asked to open their eyes. Indeed, sleep is associated with efferent vagal increases [52] and increased sleepiness has the potential to increase vagal activity (HF power). This is the opposite consequence to the noted reduction in efferent vagal tone in the SAHS subjects. The supine results were confirmed in the standing posture, which demonstrated qualitatively similar results. With this in mind, the noted reduction in HF power in the supine posture in the SAHS subjects is in all probability real, or an underestimate of the actual reduction. Inter-group differences in HF power and urine catecholamines may have been reduced as the controls were defined in terms of an $\mathrm{AHI}<10 \mathrm{~h}^{-1}$ with no symptoms of daytime sleepiness. Young et al. [53] have reported clinically significant SAHS as AHI $>5 \mathrm{~h}^{-1}$ plus symptoms and five of the 14 control subjects had AHIs $6-8 \mathrm{~h}^{-1}$. The threshold of $<10 \mathrm{~h}^{-1}$ for the controls was decided in order to obtain a greater chance of recruiting controls with higher BMIs. This may have reduced the chance of a significant difference in urine catecholamine concentrations.

\section{Conclusions}

Limited evidence of significant daytime sympathetic activation in mild sleep apnoea/hypopnoea syndrome 
subjects with a minor degree of nocturnal desaturation and no associated comorbidity was found. In the more severe (desaturating) sleep apnoea/hypopnoea syndrome group, the degree of episodic arterial oxygen saturation dips was related to waking sympathetic activity. The principal component altering sympatho-vagal balance in this mild group of sleep apnoea/hypopnoea syndrome subjects was a reduction in the waking efferent cardiac vagal tone and the decrease in vagal tone was correlated with the apnoea/ hypopnoea index. Reduced vagal tone may compromise the myocardium during periods of heightened sympathetic activity and this might contribute to the increased incidence of cardiovascular disorders in sleep apnoea/hypopnoea syndrome patients.

\section{References}

1. Marrone O, Riccobono L, Salvaggio A, Mirabella A, Bonanno A, Bonsignore MR. Catecholamines and blood pressure in obstructive sleep apnea syndrome. Chest 1993; 103: $722-727$.

2. Hedner J, Ejnell H, Sellgren J, Hedner T, Wallin G. Is high and fluctuating muscle sympathetic nerve activity in the sleep apnoea syndrome of pathogenic importance for the development of hypertension. J Hypertens 1988; 6: $529-531$.

3. Somers V, Mark A, Abboud F. Sympathetic activation by hypoxia and hypercapnia-implications for sleep apnoea. Clin Exp Hypertens 1988; 10: 413-422.

4. Narkiewicz K, van de Borne PJ, Pesek CA, Dyken ME, Montano N, Somers VK. Selective potentiation of peripheral chemoreflex sensitivity in obstructive sleep apnoea. Circulation 1999; 99: 1183 - 1189.

5. Carlson JT, Hedner J, Elam M, Ejnell H, Sellgren J, Wallin BG. Augmented resting sympathetic activity in awake patients with obstructive sleep apnoea. Chest 1993; 103: $1763-1768$.

6. Loredo JS, Ziegler MG, Ancoli-Israel S, Clausen JL, Dimsdale JE. Relationship of arousals from sleep to sympathetic nervous system activity and BP in obstructive sleep apnea. Chest 1999: 116: 655-659.

7. Narkiewicz K, Montano N, Cogliati C, et al. Altered cardiovascular variability in obstructive sleep apnea. Circulation 1998; 98: 1071-1077.

8. Stradling J, Davies RJO. Sleep apnea and hypertension-what a mess. Sleep 1997; 20: $789-793$.

9. He J, Kryger MH, Zorick FJ, Conway W, Roth T. Mortality and apnea index in obstructive sleep apnea. Experience in 385 male patients. Chest 1988; 94: 9-14.

10. Hung J, Whitford EG, Parsons RW, Hillman DR. Association of sleep apnoea with myocardial infarction in men. Lancet 1990; 336: $261-264$.

11. Burgess HJ, Trindler J, Kim Y, Luke D. Sleep and circadian influences on cardiac autonomic nervous system activity. Am J Physiol 1997; 273: H1761H1768.

12. Hartikainen $\mathbf{J}$, Tarkiainen I, Tahvanainen $\mathrm{K}$, Mantysaari M, Lansimies E, Pyorala K. Circadian variation of cardiac autonomic regulation during 24-h bed rest. Clin Physiol 1993; 13: 185- 196.

13. Levy MN, Ng M, Lipman RI, Zieske H. Vagus nerves and baroreceptor control of ventricular performance. Circ Res 1966; 18: 101-106.

14. Takahashi N, Zipes DP. Vagal modulation of adrenergic effects on canine sinus and atrioventricular nodes. Am J Physiol 1983; 244: H775-H781.

15. Schwartz PJ, Pagani M, Lombardi F, Malliani A, Brown AM. A cardiocardiac sympathovagal reflex in the cat. Circ Res 1973; 32: 215-220.

16. Muscholl E. Peripheral muscarinic control of norepinephrine release in the cardiovascular system. Am J Physiol 1980: 239: H713-H720.

17. Akselrod S, Gordon D, Ubel FA, Shannon DC, Berger $\mathrm{AC}$, Cohen RJ. Power spectrum analysis of heart rate fluctuation: a quantitative probe of beat-to-beat cardiovascular control. Science 1981: 213: 220-222.

18. Hayano J, Sakakibara Y, Yamada A, et al. Accuracy of assessment of cardiac vagal tone by heart rate variability in normal subjects. Am J Cardiol 1991; 67: 199-204.

19. Pomeranz B, Maculay RJB, Caucill MA, et al. Assessment of autonomic function in humans by heart rate spectral analysis. Am J Physiol 1985; 248: H151-H153.

20. Task Force of The European Society of Cardiology and The North American Society of Pacing and Electrophysiology. Heart rate variability: standards of measurement, physiological interpretation, and clinical use. Eur Heart $J$ 1996; 17: 354-381.

21. Malliani A, Lombardi F, Pagani M. Power spectrum analysis of heart rate variability: a tool to explore neural regulatory mechanisms. Br Heart $J$ 1994; 71: $1-2$.

22. Montano N, Ruscone TG, Porta A, Lombardi F, Pagani M, Malliani A. Power spectrum analysis of heart rate variability to assess the changes in sympathovagal balance during graded orthostatic tilt. Circulation 1994; 90: 1826-1831.

23. Appel ML, Berger RD, Saul JP, Smith JM, Cohen RJ. Beat to beat variability in cardiovascular variables: noise or music? JACC 1989; 14: 1139-1148.

24. Malliani A. The pattern of sympathovagal balance explored in the frequency domain. News Physiol Sci 1999; 14: $111-117$.

25. Noda A, Ito R, Okada T, Yasuma F, Nakashima N, Yokota M. Twenty-four-hour ambulatory oxygen desaturation and electrocardiographic recording in obstructive sleep apnea syndrome. Clin Cardiol 1998; 21: $506-510$.

26. Khoo MCK, Kim T, Berry RB. Spectral indices of cardiac autonomic function in obstructive sleep apnea. Sleep 1999; 22: 443-451.

27. Kansanen M, Vanninen E, Tuunainen A. The effect of a very low-calorie diet-induced weight loss on the severity of obstructive sleep apnoea and autonomic nervous function in obese patients with obstructive sleep apnoea syndrome. Clin Physiol 1998; 18: $377-$ 385.

28. Noda A, Yasuma F, Okada T, Yokota M. Circadian rhythm of autonomic activity in patients with obstructive sleep apnea syndrome. Clin Cardiol 1998; 21: $271-276$.

29. Roche F, Court-Fortune I, Pichot V, et al. Reduced cardiac sympathetic autonomic tone after long-term nasal continuous positive airway pressure in obstructive sleep apnoea syndrome. Clin Physiol 1999; 19: $127-134$.

30. Resta O, Rana L, Procacci V, Guido P, Picca V, Scarpelli F. Autonomic dysfunction in normotensive awake subjects with obstructive sleep apnoea syndrome. Monaldi Arch Chest Dis 1998; 53: 23-29. 
31. Cooke WH, Hoag JB, Crossman AA, Kuusela TA, Tahvanainen KU, Eckberg DL. Human responses to upright tilt: a window on central autonomic integration. J Physiol (Lond) 1999; 517: 617-628.

32. Ewing DJ, Martyn CN, Young RJ, Clarke BF. The value of cardiovascular autonomic function tests: 10 years experience in diabetes. Diabetes Care 1985; 8: $491-498$

33. Ewing DJ, Campbell IW, Murray A, Neilson JM, Clarke BF. Immediate heart-rate response to standing: simple test for autonomic neuropathy in diabetes. BMJ 1978; 1: 145- 147.

34. Kaufmann H. Investigation of cardiovascular autonomic dysfunction. In: Korczyn AD, eds. Handbook of Autonomic Nervous System Dysfunction. New York, Marcel Dekker Inc., 1995; pp. 427-468.

35. Welch PD. The use of fast Fourier transformation for the estimation of power spectra: a based on time averaging over short, modified periodograms. IEEE Trans Audio Electroacoust 1967; AU-15: 70-73.

36. Green B, Cooper JDH, Turnell DC. An automated method for the analysis of urinary free catecholamines using ASTED and high-pressure liquid chromatography. Ann Clin Biochem 1989; 26: 361-367.

37. Rechtschaffen A, Kales A. A manual of standardised terminology, techniques and scoring system for sleep stages of human subjects. Los Angeles, Brain Information service/Brain Research Institute, 1968.

38. The Atlas Task Force. EEG Arousals: Scoring rules and examples. Sleep 1992; 15: $173-184$.

39. Gould GA, Whyte KF, Rhind GB, et al. The sleep hypopnea syndrome. Am Rev Respir Dis 1988; 137: $895-898$.

40. Cortelli P, Parchi P, Sforza E, et al. Cardiovascular autonomic dysfunction in normotensive awake subjects with obstructive sleep apnoea syndrome. Clin Auton Res 1994; 4: 57-62.

41. Dimsdale JE, Coy T, Ancoli-Israel S, Mills P, Clausen J, Ziegler MG. Sympathetic nervous system alterations in sleep apnea. The relative importance of respiratory disturbance, hypoxia, and sleep quality. Chest 1997; 111: 639-642.

42. Daly MD, Angell-James JE, Elsner R. Role of carotidbody chemoreceptors and their reflex interactions in bradycardia and cardiac arrest. Lancet 1979; 1 : $764-767$.

43. Montano N, Cogliati C, Porta A, et al. Central vagotonic effects of atropine modulate spectral oscillations of sympathetic nerve activity. Circulation 1998; 98: 1394-1399.

44. Fletcher EC, Lesske J, Culman J, Miller CC, Unger T. Sympathetic denervation blocks blood pressure elevation in episodic hypoxia. Hypertension 1992; 20: $612-$ 619.

45. Fletcher EC, Lesske J, Qian W, Miller CC, Unger T. Repetitive, episodic hypoxia causes diurnal elevation of systemic blood pressure in rats. Hypertension 1992; 19: $555-561$.

46. Brooks D, Homer RL, Kozar LF, Render-Teixeira CL, Phillipson EA. Obstructive sleep apnea as a cause of systemic hypertension. Evidence from a canine model. J Clin Invest 1997; 99: 106-109.

47. O'Donnell CP, Schwartz AR, Smith PL, Robotham JL, Fitzgerald RS, Shirahata M. Reflex stimulation of renal sympathetic nerve activity and blood pressure in response to apnea. Am J Respir Crit Care Med 1996; 154: 1763 - 1770.

48. Eckberg DL. Sympathovagal balance: a critical appraisal. Circulation 1997; 96: 3224-3232.

49. Brooks D, Horner RL, Floras JS, Kozar LF, RenderTeixeira CL, Phillipson EA. Baroreflex control of heart rate in a canine model of obstructive sleep apnea. Am J Respir Crit Care Med 1999; 159: 1293 1297.

50. Eckberg DL, Drabinsky M, Braunwald E. Defective cardiac parasympathetic control in patients with heart disease. $N$ Engl J Med 1971; 285: 877-883.

51. Ferguson DW, Abboud FM, Mark AL. Selective impairment of baroreflexmediated vasoconstrictor responses in patients with ventricular dysfunction. Circulation 1984; 69: 451-460.

52. Bonnet MH, Arand DL. Heart rate variability in insomniacs and matched normal sleepers. Psychosom Med 1998; 60: 610-615.

53. Young T, Palta M, Dempsey J, Skatrud J, Weber S, Badr S. The occurrence of 51 sleep-disordered breathing among middle aged adults. $N$ Eng $J$ Med 1993; 328: $1230-1235$ 